\title{
Performance Evaluation of AODV and DSR with Reference to Network Size
}

\author{
Rajesh Deshmukh \\ Rungta College of Engineering \\ Raipur, Chhattisgarh, India
}

\author{
Asha Ambhaikar \\ Rungta College of Engineering \\ Raipur, Chhattisgarh, India
}

\begin{abstract}
A mobile ad hoc network is a collection of autonomous mobile nodes that communicate with each other over wireless links. Such networks does play important role in civilian and military settings, being useful for providing communication support where no fixed infrastructure exists or the deployment of a fixed infrastructure is possible. It is a crucial part in the performance evaluation of MANET to select suitable mobility model and routing protocols. Therefore, a number of routing protocols as well as mobility models have been proposed for ad hoc wireless networks based on different scenarios. In this paper, we study and compare the performance of the two reactive routing protocols AODV and DSR with reference to varying Network Size. For experimental purposes, we have considered increasing network size from 100 to 150 nodes and illustrate the performance of the routing protocol across Packet Delivery Ratio parameter. Our simulation result shows that both AODV \& DSR is performing equally good until the network size cross a certain limit.
\end{abstract}

\section{Keywords}

AODV, DSR, RANDOM WAYPOINT, PACKET DELIVERY RATIO, OVERHEAD

\section{INTRODUCTION}

A mobile ad-hoc network (MANET) is a collection of nodes, which have the possibility to connect on a wireless medium and form an arbitrary and dynamic network with wireless links. That means that links between the nodes can change during time, new nodes can join the network, and other nodes can leave it. A MANET is expected to be of larger size than the radio range of the wireless antennas, because of this fact it could be necessary to route the traffic through a multi-hop path to give two nodes the ability to communicate. There are neither fixed routers nor fixed locations for the routers as in cellular networks. Cellular networks consist of a wired backbone which connects the base-stations. The mobile nodes can only communicate over a one-hop wireless link to the base-station; multi-hop wireless links are not possible. By contrast, a MANET has no permanent infrastructure at all. All mobile nodes act as mobile routers. A MANET is highly dynamic. Links and participants are often changing and the quality of the links as well. Hence, a routing protocol for ad hoc networks runs on every host and is therefore subject to the limit of the resources at each mobile host. A good routing protocol should minimize the computing load on the host as well as the traffic overhead on the network. Traditional routing protocols based on the link-state or distance-vector algorithms are aimed at finding optimal routes to every host in the network, and topological changes of network can only be reflected through the propagation of periodic updates. These protocols are not suitable for ad hoc networks [2].

\section{ADHOC ROUTING PROTOCOLS}

Since the advent of Defense Advanced Research Projects Agency (DARPA) packet radio networks in the early 1970s, numerous protocols have been developed for ad hoc mobile networks. Such protocols must deal with the typical limitations of these networks, which include high power consumption, low bandwidth, and high error rates. As shown in Fig. 1, these routing protocols may generally be categorized as:

- Table-driven

- Source-initiated (demand-driven)

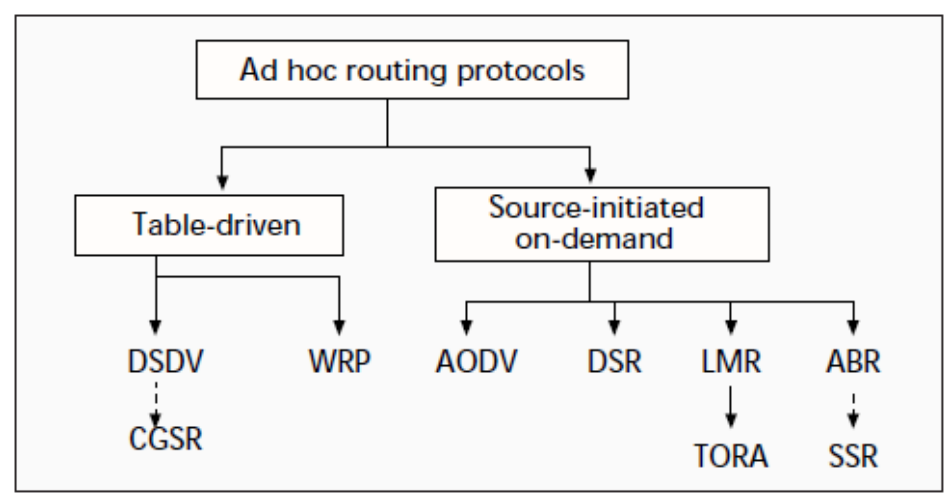

Figure 1: Classification of Ad hoc Routing Protocols

Despite being designed for the same type of underlying network, the characteristics of each of these protocols are quite distinct. The following sections describe the protocols and categorize them according to their characteristics.

\subsection{Table-Driven Routing Protocols}

Table-driven routing protocols attempt to maintain consistent, upto-date routing information from each node to every other node in the network. These protocols require each node to maintain one or more tables to store routing information, and they respond to changes in network topology by propagating updates throughout the network in order to maintain a consistent network view. The areas in which they differ are the number of necessary routingrelated tables and the methods by which changes in network structure are broadcast.

\subsection{Source-Initiated On-Demand Routing}

A different approach from table-driven routing is source-initiated on-demand routing. This type of routing creates routes only when desired by the source node. When a node requires a route to a destination, it initiates a route discovery process within the network. This process is completed once a route is found or all possible route permutations have been examined. Once a route has been established, it is maintained by a route maintenance 
procedure until either the destination becomes in accessible along every path from the source or until the route is no longer desired.

\subsection{Ad hoc On-demand Distance Vector Routing (AODV)}

AODV is an on-demand protocol, which initiate route request only when needed. When a source node needs a route to certain destination, it broadcasts a route request packet (RREQ) to its neighbors. Each receiving neighbor checks its routing table to see if it has a route to the destination. If it doesn't have a route to this destination, it will re-broadcast the RREQ packet and let it propagate to other neighbors. If the receiving node is the destination or has the route to the destination, a route reply (RREP) packet will be sent back to the source node. Routing entries for the destination node are created in each intermediate node on the way RREP packet propagates back. A hello message is a local advertisement for the continued presence of the node. Neighbors that are using routes through the broadcasting node will continue to mark the routes as valid. If hello messages from a particular node stop coming, the neighbor can assume that the node has moved away. When that happens, the neighbor will mark the link to the node as broken and may trigger a notification to some of its neighbors telling that the link is broken. In AODV, each router maintains route table entries with the destination IP address, destination sequence number, hop count, next hop ID and lifetime. Data traffic is then routed according to the information provided by these entries [4].

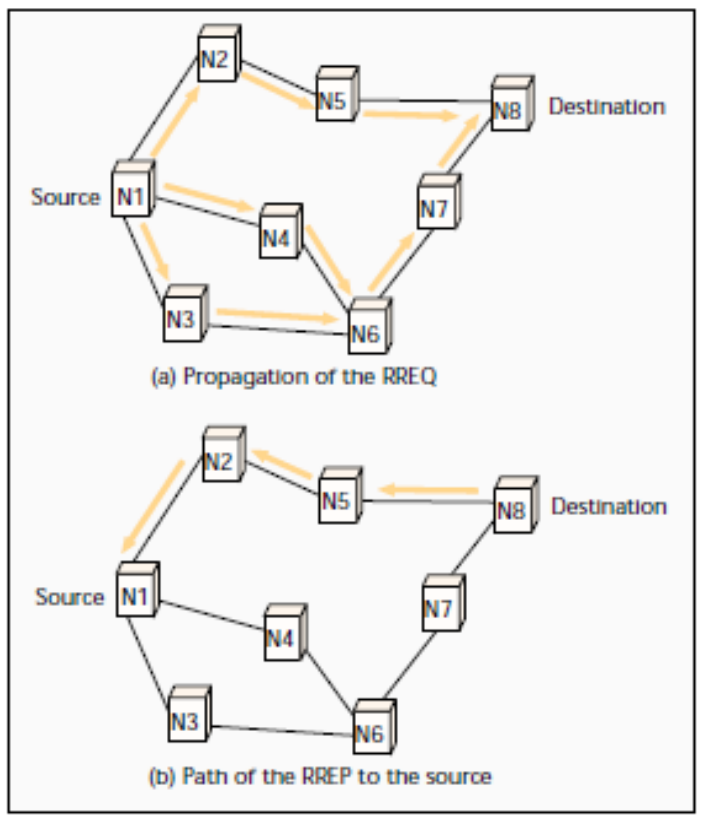

Figure 2: AODV route discovery

\subsection{Dynamic Source Routing (DSR)}

Dynamic Source Routing protocol is a reactive protocol i.e. it determines the proper route only when a packet needs to be forwarded. The node floods the network with a route-request and builds the required route from the responses it receives. DSR allows the network to be completely self-configuring without the need for any existing network infrastructure or administration.
The DSR protocol is composed of two main mechanisms that work together to allow the discovery and maintenance of source routes in the ad hoc network. All aspects of protocol operate entirely on-demand allowing routing packet overhead of DSR to scale up automatically. Route Discovery: When a source node S wishes to send a packet to the destination node $\mathrm{D}$, it obtains a route to D. This is called Route Discovery. Route Discovery is used only when $\mathrm{S}$ attempts to send a packet to $\mathrm{D}$ and has no information on a route to D. Route Maintenance: When there is a change in the network topology, the existing routes can no longer be used. In such a scenario, the source $S$ can use an alternative route to the destination $\mathrm{D}$, if it knows one, or invoke Route Discovery. This is called Route Maintenance [6] [7].

\section{MOBILITY MODELS}

\subsection{Random Waypoint Mobility Model}

To evaluate the performance of a protocol for an adhoc network, it is necessary to test the protocol under realistic conditions, especially including the movement of the mobile nodes. Since not many MANETs have been deployed, most of this research is simulation based. These simulations have several parameters including the mobility models and the communicating traffic pattern. MANET protocol performance may vary drastically across different mobility models. In the literature, there are a lot of models used, mostly in simulations. Among the common one is the Random Waypoint Model, which is a simple model that may be applicable to some scenarios. However, there are other mobility models that may be used to capture the more important mobility characteristics of scenarios that MANETs may develop.

A mobile node begins the simulation by waiting a specified pause time. After this time it selects a random destination in the area and a random speed distributed uniformly between $0 \mathrm{~m} / \mathrm{s}$ and Vmax. After reaching its destination point, the mobile node waits again pause time seconds before choosing a new way point and speed. The mobile nodes are initially distributed over the simulation area. This distribution is not representative to the final distribution caused by node movements. To ensure a random initial configuration for each simulation, it is necessary to discard a certain simulation time and to start registering simulation results after that time.

The Random Waypoint Mobility Model is very widely used in simulation studies of MANET. As described in the performance measures in mobile ad hoc networks are affected by the mobility model used. One of the most important parameters in mobile ad hoc simulations is the nodal speed. The users want to adjust the average speed to be stabilized around a certain value and not to change over time. They also want to be able to compare the performance of the mobile ad hoc routing protocols under different nodal speeds. For the Random Waypoint Mobility Model a common expectation is that the average is about half of the maximum, because the speeds in a Random Waypoint Model are chosen uniformly between $0 \mathrm{~m} / \mathrm{s}$ and $V \max$.

The average speed decreases over time and will approach 0 . This could lead to wrong simulation results. This phenomenon can be intuitively explained as follows. In the Random Waypoint Mobility Model a node selects its destination and its speed. The node keeps moving until it reaches its destination at that speed. If it selects a far destination and a low speed around $0 \mathrm{~m} / \mathrm{s}$, it travels 
for a long time with low speed. If it selects a speed near Vmax the time traveling with this high speed will be short. After a certain time the node has traveled much more time at low speed than at high speed. The average speed will approach $0 \mathrm{~m} / \mathrm{s}$. The suggestion in to prevent this problem is choosing, e.g. $1 \mathrm{~m} / \mathrm{s}$ instead of $0 \mathrm{~m} / \mathrm{s}$ as Vmin. With this approach the average speed stabilizes after a certain time at a value below 1/2 Vmax.

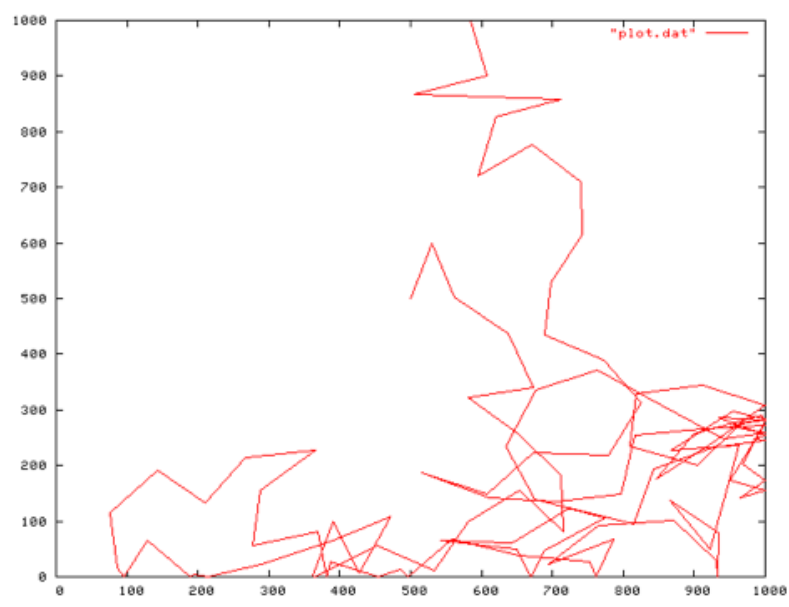

Figure 3: Travelling pattern of an MN using the Random Waypoint Mobility Model

\subsection{Random Direction Mobility Model}

To reduce density waves in the average number of neighbors by the Random Waypoint Model the Random Direction Mobility Model was created. Density waves are the clustering of nodes in one part of the simulation area. For the Random Waypoint Mobility Model the probability of choosing a location near the center or a way point which requires traveling through the center of the area is high. The Random Direction Mobility Model was invented to prevent this behavior and to promote a semi constant number of neighbors. The mobile node selects a direction and travels to the border of the simulation area. If the boundary is reached, the node pauses for a specific time and then chooses a new direction and the process goes on. Because of pausing on the border of the area, the hop count for this mobility model is much higher than for most other mobility.

\subsection{Reference Point Group Mobility Model}

In reference point group mobility model, simulate group behavior, where each node belongs to a group where every node follows a logical centre (group leader) that determines the group's motion behavior. The nodes in a group are usually randomly distributed around the reference point. The different nodes use their own mobility model and are then added to the reference point which drives them in the direction of the group. At each instant, every node has a speed and direction that is derived by randomly deviating from that of the group leader. This general description of group mobility can be used to create a variety of models for different kinds of mobility applications. Group mobility as such can be used in military battlefield communications.

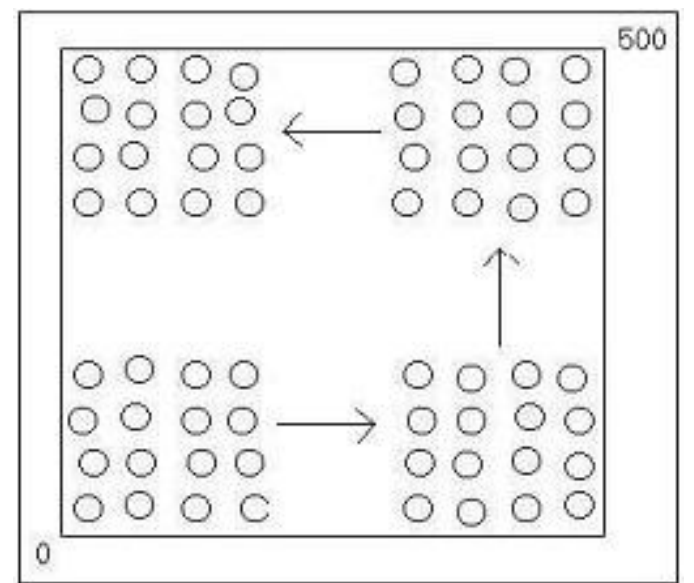

Figure 4: Grouping of MN in Reference Point Group Mobility Model

In following figure, four MNs are initially placed in the lower lefthand corner of the simulation area. A black square is the group center; the circles near the group center are MNs in the group. One circle in figure is gray in order to distinguish it from the other MNs in the group. RPGM first calculates each MN's reference point using the group motion vector GM that may be randomly chosen or predefined. The current reference point of the gray MN, t RP, moves towards the right hand corner of the simulation area alongside the group center. This location becomes the (new reference point, t RP +1 ), for the gray MN. Finally, the new position for the gray $\mathrm{MN}$ is calculated by summing a random motion vector, RM, with the new reference point. The length of $\mathrm{RM}$ is uniformly distributed within a specified radius centered at $(t \mathrm{RP}+1)$ and its direction is uniformly distributed between 0 and $2 \pi$. This process is repeated for each $\mathrm{MN}$ in the group. The RPGM model was designed to depict scenarios such as an avalanche rescue. During an avalanche rescue, the responding team consisting of human and canine members work cooperatively. The human guides tend to set a general path for the dogs to follow, since they usually know the approximate location of victims. The dogs each create their own "random" paths around the general area chosen by their human counterparts. If appropriate group paths are chosen, along with proper initial locations for various groups, many different mobility applications may be represented with the RPGM model.

\subsection{Manhattan Grid Mobility Model}

The Manhattan mobility model is proposed to model movement in an urban area. In the Manhattan model, the mobile node is allowed to move along the horizontal or vertical streets on the urban map. At an intersection of a horizontal and a vertical street, the mobile node can turn left, right or go straight. The probability of moving on the same street is 0.5 , the probability of turning left is 0.25 and the probability of turning right is 0.25 . The velocity of a mobile node at a time slot is dependent on its velocity at the previous time slot. Also, a node's velocity is restricted by the velocity of the node preceding it on the same lane of the street. Manhattan mobility model focuses on nodes moving along horizontal or vertical streets, which is not enough to model nodes moving along non-horizontal and non-vertical streets. Moreover, Manhattan model is not suitable to model the movement happening in the intersections of highway systems, this is much more complex than the intersection of local streets. Thus, 
Manhattan mobility model is expected to have high spatial dependence and high temporal dependence. On top of that, it also imposes geographic restrictions on node mobility, though it gives flexibility for the nodes to change its direction.

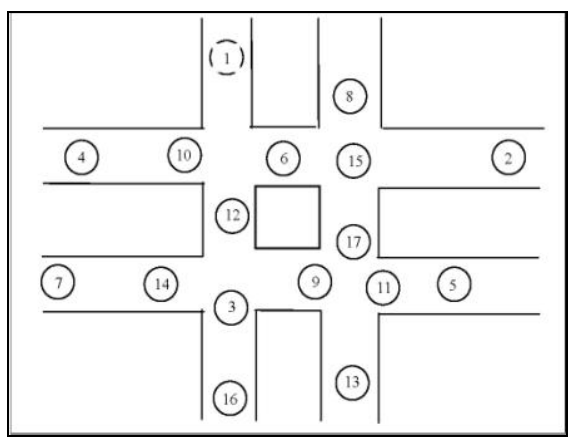

Figure 5: Topography showing the movement of nodes for Manhattan mobility model

\subsection{Gauss Markov Mobility Model}

In Gauss Markov Model, for each mobile node two separate values are maintained instead of one speed vector: The mobile's speed and its direction of movement. The default method of handling mobile nodes that move out of the simulation is that nodes may continue to walk beyond the area boundary, which causes the next movement vector update not to be based on the prior angle, but on an angle that brings the nodes back onto the field. Therefore, the field size is automatically adapted to the node movements after scenario generation. New speed and direction of movement are simply chosen from a normal distribution with a mean of the respective old. Speed values are constrained to a certain interval that can be specified if a newly chosen speed value is outside of this interval, it is changed to the closest value inside of the interval (which is either the minimum or the maximum value).

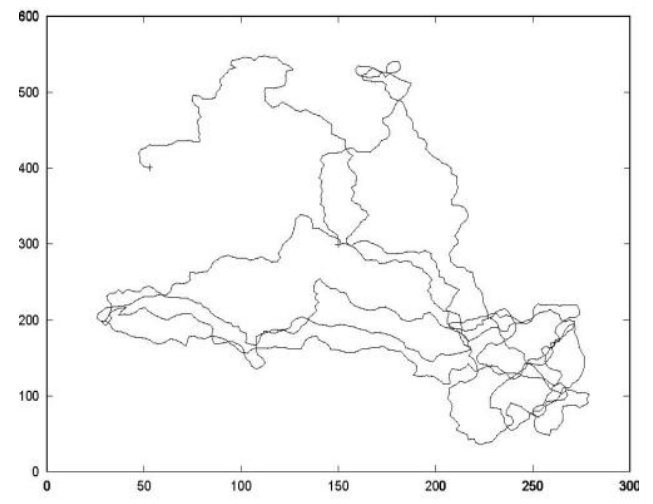

Figure 6: Travelling pattern of MN using Gauss-Markov Mobility Model

\section{THE TRAFFIC AND SCENARIO GENERATOR}

Continuous bit rate (CBR) traffic sources are used. The sourcedestination pairs are spread randomly over the network. The mobility model uses Random Waypoint mobility model in a 1020 m x 1020 m field with network load of 4 packet/s whereas network size is varied from 100 nodes to 150 nodes. Here, each packet starts its journey from a random location to a random destination with a randomly chosen speed. Once the destination is reached, another random destination is targeted after a pause. The pause time, which affects the relative speeds of the mobile hosts, is kept constant at $10 \mathrm{~s}$. Simulations are run for 100 simulated seconds whereas Maximum speed is $10 \mathrm{~m} / \mathrm{s}$.

\section{PERFORMANCE METRICS \\ Following important metrics are evaluated-}

Packet Delivery ratio (PDR) - Packet delivery ratio is calculated by dividing the number of packets received by the destination through the number of packets originated by the CBR source.

Loss Packet Ratio (LPR) - Loss Packet Ratio is calculated by dividing the number of packets that never reached the destination through the number of packets originated by the CBR source.

Routing Overhead - Routing overhead, which measures the ratio of total routing packets sent and the total number of packets sent.

\section{SIMULATION SETUP}

Simulation has been carried out by Network Simulator 2.33. In our simulation, we have used network load at the rate of 4 packets / s. Network size is increased gradually from $100-150$ nodes with constant pause time 10s.

In this simulation we wanted to investigate how the protocol behaves with increasing network size.

Table 1. Evaluation with Varying Network Size

\begin{tabular}{|c|c|}
\hline Parameter & Value \\
\hline Protocols & AODV, DSR \\
\hline Simulation Time & $100 \mathrm{~s}$ \\
\hline Number of Nodes & $100,110,120,130,140,150$ \\
\hline Network Load & 4 Packets / sec \\
\hline Pause Time & $10 \mathrm{~s}$ \\
\hline Environment Size & Constant Bit Rate \\
\hline Traffic Type & $10 \mathrm{~m} / \mathrm{s}$ \\
\hline Maximum Speed & Random Waypoint \\
\hline Mobility Model & NS 2.33 \\
\hline Network Simulator & \\
\hline &
\end{tabular}

\section{RESULTS AND DISCUSSIONS}

During the simulation we have increased the network size and recorded the performance of both the protocols. We did this simulation for 100 secs with maximum 8 cbr connections. Readings were taken for different network sizes (100, 110, 120, 130, 140 and 150 nodes). From the results it is evident that as the network size increases the Packet Delivery ratio decreases, Loss Packet Ratio Increases and Routing Overhead increases.

\subsection{PERFORMANCE EVALUATION}

Throughout the simulation performance of AODV is consistent and is always on the way of improvement, even though it is not as 
good as compared to the performance of DSR. It can be observed in figure 7 , that Packet delivery ratio performance of ADOV improves gradually as the number of nodes increases. Although PDR both AODV and DSR is dropped drastically at the same point when number of nodes are between 110 to120. Improvement in DSR is observed when number of nodes exceeds 120, while improvement in AODV is observed as the number of nodes reached 130. AODV starts to perform much better than DSR when the number of nodes exceeds 135 nodes, while DSR shows a big drop in the PDR.

It can be observed in figure 8, that Loss Packet Ratio in AODV is always greater as compared to DSR, even though it can be observed that performance of AODV is drastically improved as the number of nodes exceeds 140, while DSR starts to perform poorly at the same point.

After observing in figure 9, AODV and DSR are performing equally in terms of Routing Overhead factor. Even though DSR performs much better as compared to AODV, until a point where number of nodes exceeds 140. Around 115 nodes value, performance of both AODV and DSR starts to improve. AODV continues to improve while DSR shows more routing overhead.

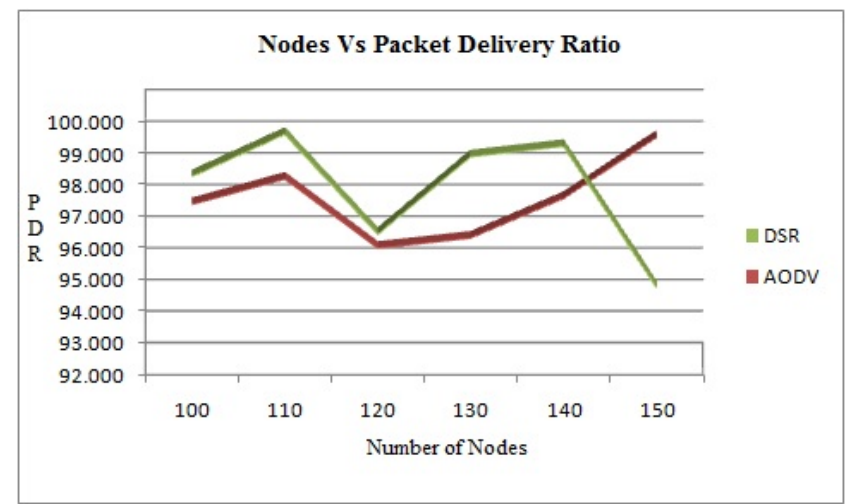

Figure 7: Number of Nodes Vs Packet Delivery Ratio

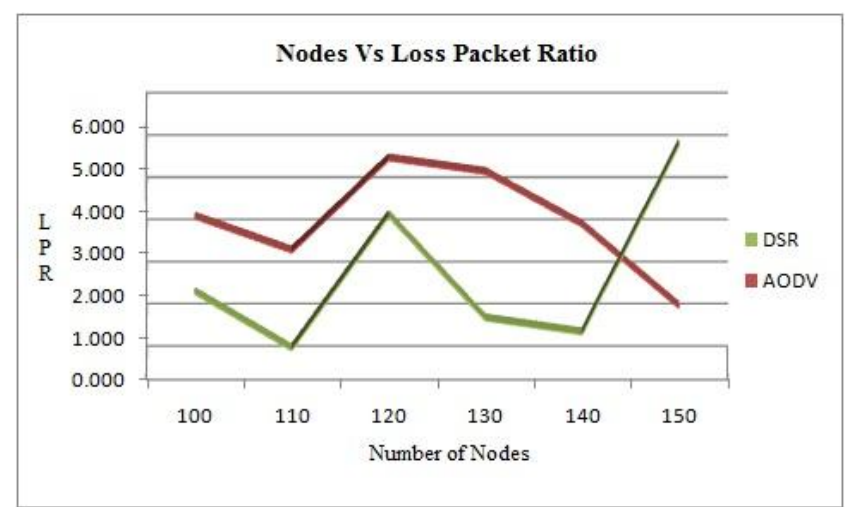

Figure 8: Number of Nodes Vs Loss Packet Ratio

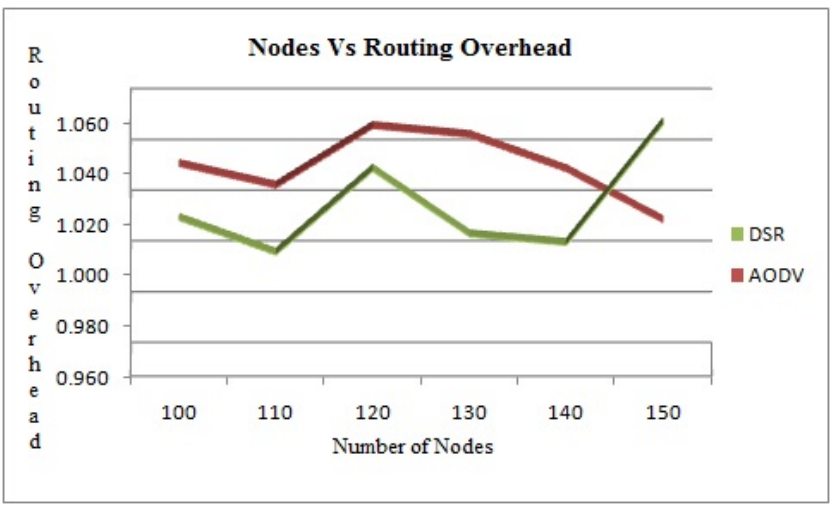

Figure 9: Number of Nodes Vs Routing Overhead

\section{CONCLUSION AND FUTURE WORK}

Empirical results illustrate that the performance of a routing protocol varies widely across different network sizes and hence the study results from one scenario cannot be applied to other scenario. Hence we have to consider the network size of an application while selecting a routing protocol. Our simulation results have given an indication that AODV performs better on larger number of nodes with given scenario while, DSR is performs better on lesser number of nodes with the same scenario.

The future scope is to find out what factors are responsible for these simulation results, as performance of AODV in various situations as compared to DSR are not as expected. Further simulation needs to be carried out for the performance evaluation with not only increased number of nodes but also varying other related parameters like Pause Time, Network load, Speed, Mobility modes etc.

\section{REFERENCES}

[1] Asha Ambhaikar and Lokesh Kumar Sharma, "Exploring the Behaviour of Mobile Ad hoc Network Routing Protocols with Reference to Speed and Terrain Range", IMECS 2010, Volume II, March 17 - 19, 2010, ISBN: 978-988-18210-4-1

[2] Charles E. Perkins, Elizabeth M. Royer, "Ad hoc On Demand Distance Vector Routing Protocol"

[3] Elizabeth M. Royer, C.K. Toh, "A Review of Current Routing Protocols for Ad Hoc Mobile Wireless Networks"

[4] Aditya Goel, Ajaii Sharma, "Performance Analysis of Mobile Ad-hoc Network Using AODV Protocol", International Journal of Computer Science and Security (IJCSS), Volume (3): Issue (5), Page No.334

[5] Yasser Kamal Hassan, Mohamed Hashim Abd El-Aziz, and Ahmed Safwat Abd El-Radi , "Performance Evaluation of Mobility Speed over MANET Routing Protocols", International Journal of Network Security, Vol.11, No.3, PP.128\{138, Nov. 2010, Page No.128

[6] Mamoun Hussein Mamoun, "Performance Comparison of Mobile Ad-hoc Network Routing Protocol”, MCN 2007 Conference, November 7-10, 2007 , Page No.07. 
[7] A.Boomarani Malany, V.R.Sarma Dhulipala, and RM.Chandrasekaran, "Throughput and Delay Comparison of MANET Routing Protocols", Int. J. Open Problems Compt. Math., Vol. 2, No. 3, September 2009 , ISSN 19986262, Page No.461

[8] Ian D. Chakeres, Elizabeth M. Belding-Royer, "AODV Routing Protocol Implementation Design",

[9] Stuart Kurkowski, Tracy Camp, Michael Colagrosso, "MANET Simulation Studies: The Incredibles", Mobile Computing and Communications Review, Volume 1, Number 2, Page No.01

[10] Yu-Doo Kim, Il-Young Moon, Sung-Joon Cho, "A comparison of improved AODV routing protocol based on IEEE 802.11 AND IEEE 802.15.4”, Journal of Engineering Science and Technology Vol. 4, No. 2 (2009) 132 - 141, Page No.132

[11] Natarajan Meghanathan, "Impact of the Gauss-Markov Mobility Model on Network Connectivity, Lifetime and Hop Count of Routes for Mobile Ad hoc Networks", JOURNAL OF NETWORKS, VOL. 5, NO. 5, MAY 2010, Page No.509

[12] Nadia Qasim, Fatin Said, Hamid Aghvami, "Mobile Ad Hoc Networking Protocols' Evaluation through Simulation for Quality of Service", IAENG International Journal of Computer Science, 36:1, IJCS_36_1_10

[13] Petteri Kuosmanen, "Classification of Ad Hoc Routing Protocols".

[14] Neeti Soni, "Exploiting the need of Comparative study of routing protocols and Misbehaving node in wireless network", Published in International Journal of Advanced Engineering \& Application, June 2010 Issue

[15] Anuj K. Gupta, Dr. Harsh Sadawarti, Dr. Anil K. Verma, "Performance analysis of AODV, DSR \& TORA Routing Protocols", IACSIT International Journal of Engineering and Technology, Vol.2, No.2, April 2010, ISSN: 1793-8236

[16] Ian D. Chakeres, Luke Klein-Berndt, "AODVjr, AODV Simplified", Mobile Computing and Communications Review, Volume 6, Number 3

[17] UCB/LBNL/VINT Network Simulator, http://wwwmash.cs.berkeley.edu/ns/.
[18] E.L. Madruga, J.J. Garcia -Luna-Aceves. Scalable Multicasting: The Core- Assisted Mesh Protocol. 1999.

[19] M.W. Subbarao: Mobile Ad Hoc Data Networks for Emergency Preparedness Telecommunications - Dynamic Power-Conscious Routing Concepts. Submitted as an interim project for Contract Number DNCR086200 to the National Communications $\quad$ Systems. 2000 http://www.w3.antd.nist.gov/ subbarao/MANET/manet.html

[20] S.R.Das, R Castaneda. J Yan. Comparative Performance Evaluation of Routing Protocols for Mobile, Ad hoc Networks. http://www.ececs.uc.edu/ sdas/pub.html

[21] Prabhakar Dhekne, "Wireless Sensor Network", Talk at SASTRA, Bhabha Atomic Research Centre, August 24, 2006

[22] "The Network Simulator - ns-2," available at http://www.isi.edu/nsnam/ns/.

[23] Rainer Baumann,, AODV, Presentation at ETH Zürich, April 2002

[24] http://www.ee.surrey.ac.uk/Personal/G.Aggelou/PAPERS/ma druga.monet99.pdf

[25] C-K Toh. Ad Hoc Mobile Wireless Networks, Protocols and systems. Prentice Hall PTR. 2002. ISBN 0-13-007817-4.

[26] Jiang X, Camp T: “A review of Geocasting Protocols for a Mobile Ad Hoc Network, Grace Hopper Celebration (GHC), 2002, http://toilers.mines.edu/papers/

[27] M.W. Subbarao: "Dynamic Power-Conscious Routing for MANET:s An Initial Approach", Proceeding of IEEE VTC Fall 1999, Amsterdam, The Netherlands, 1999. http://www.nvl.nist.gov/pub/nistpubs/jres/104/6/j46sub.pdf

[28] M.W. Subbarao: Ad Hoc Networking Critical Features and Performance Metrics, 1999. http://w3.antd.nist.gov/pubs99.shtml

[29] M.S. Corson and J. Macker. Mobile Ad Hoc Networking (MANET): Routing Protocol Performance issues and Evaluation Considerations. Request for Comments 2501, IETF, January 1999. http://www.rfc.net/rfc2501.html

[30] Trace graph website http://www. geocities.com/tracegraph 